\title{
Perfil de un grupo de audiencia universitaria en México según su participación ciudadana, confianza en las instituciones e interacción cívica en los medios en 2017
}

\author{
Profile of a group of university audience in Mexico \\ according to their citizen participation, trust in \\ institutions and civic interaction in the media in 2017
}

\author{
Gutiérrez-Rentería, M. E., Santana, J. C., López-Hernández, C., Pérez- \\ Ayala Madero, M. G. y Hellmueller, L. ${ }^{1}$ \\ Recibido el 23 de marzo de 2018 - Aceptado el 14 de junio de 2018 \\ DOI: https://doi.org/10.26441/RC17.2-2018-A5
}

\begin{abstract}
RESUMEN: Esta investigación de enfoque cuantitativo muestra las singularidades de una audiencia universitaria en México en 2017, medida según su participación cívica, la valoración que da a las instituciones y la actuación interactiva que tiene con los medios respecto a temas sociales y políticos. La población encuestada estuvo formada por 1,195 jóvenes adultos de 23 años de edad promedio y que forma parte de la comunidad universitaria situada en Jalisco, Ciudad de México y Aguascalientes. Los resultados muestran que la participación offline de capital social fue más significativa que la online. Las instituciones que tuvieron índice aprobatorio fueron las religiosas, las universidades y los medios de comunicación. En este ámbito, existieron diferencias significativas al evaluarlas entre grupos de edad y según el sexo. Esta audiencia tuvo rasgos de ser pasiva y conservadora respecto a su consumo informativo y reflejó apatía para manifestar su opinión en los medios de comunicación del país.
\end{abstract}

Palabras clave: audiencia universitaria; capital social; confianza.

ABSTRACT: The present quantitative study shows the singularities of the university audience in Mexico in 2017, measured according to their civic participation, how they assess national institutions, and how they interact with the media regarding social and political issues. The surveyed population

\footnotetext{
María-Elena Gutiérrez-Rentería es Doctora en Comunicación Pública por la Universidad de Navarra y Profesora Investigadora de la Universidad Panamericana. egutierr@up.edu.mx, https://orcid.org/0000-00015431-8223

Josefina C. Santana es Doctora en Educación por el ITESO, Universidad Jesuita de Guadalajara y Profesora Investigadora de la Universidad Panamericana. jsantana@up.edu.mx, https://orcid.org/0000-0001-7923-5088

Carlos López-Hernández es Doctor en Dirección de las Organización por la Universidad Popular Autónoma del Estado de Puebla y Profesor investigador de la Universidad Panamericana. calopez@up.edu.mx, https://orcid. org/0000-0003-3054-9670

Martha-Guadalupe Pérez-Ayala Madero es Doctora en Educación por la Universidad Autónoma de Guadalajara y Profesora Investigadora de la Universidad Panamericana. mperez@up.edu.mx, https://orcid.org/0000-00017399-8674

Lea Hellmueller es Doctora en Periodismo por la Universidad de Fribourg (Suiza) y Profesora Asistente de Periodismo de la Valenti School of Communication de la Universidad de Houston (Estados Unidos). Ichellmu@ Central.UH.EDU, https:// 0000-0002-6609-9395
} 
consisted of 1,195 young adults of 23 years of average age and who are part of private universities located in Jalisco, Mexico City and Aguascalientes. The results indicate that the offline participation in social capital is more significant than online participation of this university audience. The only institutions that have an approval index for this audience are religious institutions, universities, and the media. Finally, the university audience in Mexico has features of being passive and conservative regarding its consumption of information and its political participation in media. This population reflects apathy in expressing their opinion in media regarding social and political issues in the country.

Keywords: university audience; social capital; trust.

\section{Introducción}

El uso de medios de comunicación implica la creación de nuevas formas de acción e interacción en el mundo social. También, el uso de estos medios y el consumo de noticias puede llevar a transformar la organización temporal de las instituciones y la forma de interactuar de las personas en su vida cotidiana y social (Thompson, 1995). Desde la mirada de las variables microeconómicas de la industria de medios, la sociedad debe ser considerada como la audiencia objetivo a la que el empresario de la comunicación dirija estrategias para ganarse su lealtad a través de la oferta informativa que realiza. De esta forma, la confianza de la audiencia contribuye a fortalecer el valor de la marca en el mercado de la información y, a su vez, a la construcción de capital social del país en el que opera la empresa.

El objetivo de esta investigación es identificar el perfil de un grupo dentro de la audiencia universitaria en México con base en su participación política y ciudadana; la confianza que tiene en las instituciones, y el nivel de interactividad con los medios de comunicación. El método de investigación es de índole cuantitativo.

Actualmente, la población mexicana está conformada por cerca de 120 mi- llones de personas y la edad mediana es de 27 años, según el último censo de población realizado por el Instituto Nacional de Estadística y Geografía (INEGI) en 2015 (INEGI, 2016). Tener estudios universitarios en este país aún se considera un privilegio. El porcentaje de la población que tiene más de quince años con instrucción superior es cerca del $19 \%$ según los datos oficiales registrados (INEGI, 2016). Mientras que el estudio "Panorama de la Educación" realizado por la Organización para la Cooperación y el Desarrollo Económico (OCDE) muestra que en 2016 sólo el $17 \%$ de la población de entre 25 a 64 años de edad tiene grado de educación superior en esta región (OCDE, 2017).

\section{Antecedentes}

Las circunstancias macro y microeconómicas que han sorteado los empresarios de la industria de la comunicación son distintas según los periodos históricos del país y, de alguna forma, también han repercutido en la cultura democrática y participación ciudadana. En México, al igual que en el resto de los países latinoamericanos, el sistema político se ha caracterizado por la carencia de una tradición democrática. La relación entre el partido político gobernante, la forma de 
gobierno, los grupos empresariales y los medios de comunicación repercutió directamente en la política, economía, cultura y sociedad mexicanas (GutiérrezRentería, 2011).

Por otra parte, México también se enfrenta a una nueva dinámica competitiva en el mercado de la información. Esto como resultado de la liberalización de la industria, las tecnologías digitales y el cambio en los hábitos de consumo de medios por parte de la sociedad. Tradicionalmente, la radio y televisión comercial han sido los medios de mayor poder de influencia en la opinión pública, y también son los mismos que acaparan la mayoría de la inversión publicitaria. Actualmente, la oferta informativa se ha incrementado debido al surgimiento de los medios nativos digitales (Gutiérrez-Rentería, 2017), los mismos que han sabido ganarse la lealtad del público. Por otro lado, las redes sociales en internet son la principal fuente de acceso a las noticias. Las más utilizadas son Facebook, Youtube, WhatsApp y Twitter.

Los estudios sobre los jóvenes universitarios y su participación política y ciudadana a través de los medios se han incrementado en la última década. Puede considerarse que las elecciones presidenciales de 2012 influyeron en el aumento del número de investigaciones académicas en este país.

Al respecto, Domínguez, López y Ortiz-Henderson (2017) realizaron un meta-estudio titulado "Redes sociales digitales y participación política de jóvenes universitarios de México y Chile: Una revisión de estudios" en el que identificaron doce investigaciones mexicanas realizadas entre 2011 y 2016. Cinco de éstas estuvieron dedicadas al estudio del comportamiento cívico de los jóvenes de la Ciudad de México, debido al movimiento universitario \#Yosoy132 (Galindo y González-Acosta, 2013; Martínez y Acosta, 2016; Meneses, Ortega y Urbina, 2014; Ortiz y Nájera, 2014; Portillo, 2014). Existen otros estudios realizados por Domínguez et al. (2017) que tratan el tema de la participación ciudadana en este mismo segmento de edad, pero fueron realizadas en distintos estados de la República Mexicana (Aguirre, Casco y Zavariz, 2016; Farías, 2014; Leyva, Muñiz y Flores, 2015; Padilla, 2014).

Estas investigaciones utilizan distintas metodologías y tamaños de muestra. No obstante, se considera importante mencionar los principales hallazgos encontrados por estos académicos que contribuyen al objeto de estudio de esta investigación.

Por ejemplo, Galindo y GonzálezAcosta (2013) mostraron que el 58\% de sus jóvenes encuestados entró en la categoría de "pesimistas agobiados", como resultado del desánimo y hartazgo del periodo electoral en 2012. Mismo estado de ánimo que encontraron Martínez y Acosta (2016), quienes describen a la comunidad de jóvenes como "una comunidad reflexiva, 'resistencial' y anti-sistémica” (Martínez y Acosta, 2016, p. 95) que se suma a la conversación nacional a través de las redes sociales en internet. Farías (2014) encontró este mismo tipo de comportamiento de la comunidad juvenil 
mexicana y su participación política en las redes sociales.

Por su parte, Meneses, Ortega y Urbina (2014) encontraron que más del $80 \%$ de los encuestados dijo tener participación cívica de regular a muy alta, aunque dicha participación era ajena a los partidos políticos y sólo el 15\% de los jóvenes afirmó que estaba involucrado en asuntos comunitarios. Otros autores como Aguirre, Casco y Zavariz (2016), Padilla (2014), Portillo (2014) y Reguillo (2012) coinciden en los resultados de sus investigaciones respecto a que la participación cívica de los jóvenes en internet no estaba vinculada a los partidos políticos. No obstante, Macías (2014) argumenta en su investigación cómo las redes sociales en internet generan capital social en México. Este estudio coincide con los hallazgos encontrados posteriormente por Leyva, Muñiz y Flores (2015).

Por otra parte, han sido pocos los estudios científicos que tratan sobre las características de las audiencias de medios medidas por su nivel de segmentación en México y por el tipo de consumo informativo que tienen. $\mathrm{Al}$ respecto, se considera pertinente mencionar en particular a dos, que por su naturaleza de estudio también están vinculados al objeto de esta investigación.

El primer trabajo académico es el titulado: Smartphone: usos y gratificaciones de los jóvenes en México en 2015, de las autoras Gutiérrez-Rentería, SantanaVillegas y Pérez-Ayala (2016). El estudio muestra que el smartphone es el medio que más utilizan los jóvenes adultos para informarse y entretenerse, siendo
WhatsApp la red social más importante para interactuar con la familia y amigos. El $71 \%$ de los jóvenes prefieren manifestar sus opiniones y dialogar en persona en lugar de hacerlo a través del uso de medios y de redes sociales.

El segundo estudio que ayuda a comprender las características de la audiencia global altamente consumidora de noticias en medios, es el realizado por Gutiérrez-Rentería (2017). En la investigación titulada "Digital News Report 2017", en el apartado dedicado a México, evidencía que el $49 \%$ de la población confiaba en el sistema periodístico del país, mientras que el 55\% tenía mayor confianza en las noticias difundidas por las empresas de comunicación que buscaban activamente.

\section{Marco teórico}

Este apartado abarca dos ámbitos de la literatura que son necesarios para cumplir con el objetivo del estudio. El primero recopila los principales hitos relacionados al objetivo de los empresarios de la comunicación desde la mirada del media management. El segundo compila los principales elementos que aportan académicos que estudian el tema del capital social y el vínculo que existe para la construcción del mismo a través de la participación ciudadana en medios.

\subsection{La naturaleza del producto infor- mativo y la responsabilidad social del empresario}

En diversos foros académicos y de la industria, se ha estudiado el vínculo estrecho que existe entre los medios de comunicación y los partidos políticos de 
una sociedad. Según Havens y Lotz (2012), históricamente se ha considerado al trabajo periodístico de los empresarios de la comunicación como fruto de la representación de un partido político, más que una preocupación por la objetividad de las noticias y del público al que se compromete.

Sin embargo, el ciudadano -llámese consumidor, usuario o audiencia- debe considerarse como el objetivo principal de los empresarios de la comunicación y de los anunciantes que participan en la economía de medios (Albarran, 2017). Por ello, uno de los activos más valiosos para el empresario es la creación de la lealtad hacia la marca informativa al ganarse la confianza de las audiencias y los anunciantes.

Respecto a este tema de la lealtad, Rinsdorf (2017) dice que la semántica de la marca de la empresa de comunicación se basa, también, en la confianza. El nivel de confianza de los ciudadanos hacia los medios depende de la medida en que las noticias de las empresas de comunicación cumplen con las expectativas de la audiencia en reportar información veraz además de actuar como guardianes de la democracia (Rinsdorf, 2017: 227). Este autor conceptualiza confianza como el nivel de certidumbre que tiene la audiencia en el sistema periodístico de un país.

En este mismo sentido, Picard (2014) y Sánchez-García (2016) advierten que las decisiones personales e independientes hechas por los individuos como miembros de la audiencia, usuarios o consumidores, tienen efectos colectivos que repercuten en la generación de ca- pital social. Estas consecuencias pueden verse en la configuración de grupos de audiencias con formas particulares respecto al uso de los medios de comunicación.

Anteriormente, se consideraba a la audiencia como un público masivo. Albarran (2017), Gimpel (2015) y Picard (2014) coinciden en que los empresarios de la comunicación ven ahora de forma distinta al público objetivo, resultado de la fragmentación de audiencias y a las diversas formas de acceso a contenidos que tienen. Las características de las audiencias, según sus hábitos y gastos destinados al consumo de información y entretenimiento, pueden identificarse y clasificarse según el ciclo de vida, grupos de edad y etnicidad en la que se encuentran las distintas generaciones (Albarran, 2017).

Doyle (2013) señala que la fragmentación de las audiencias masivas y el empoderamiento progresivo de las personas para expresar sus preferencias a través de las plataformas digitales, han modificado los modos de interacción entre la oferta y la demanda en la industria. Para esta autora, al igual que para Sylvie (2008), la consolidación de la ventaja competitiva del empresario de la comunicación consiste también en lograr la segmentación eficaz del mercado; así como en hacer énfasis en el mercado meta al que se dirige el contenido según las características específicas de los grupos de audiencias (Doyle, 2013).

En su estudio "Las industrias informativas: ¿tienen futuro?”, Picard (2014) divide a las audiencias en cuatro tipos, según las características de consumo: 
las audiencias pasivas, las conservadoras, las creadoras, y las absorbentes.

Las audiencias pasivas prefieren leer periódicos, revistas y libros, además de ver televisión abierta. Este tipo de audiencias desempeñan el rol de receptores en la comunicación y tienen poca interacción con el medio o con otra persona a través del medio escogido. Por su parte, las audiencias que activamente consumen contenidos mediáticos pueden ser conceptualizadas como conservadoras (utilizan prioritariamente voz, mensajes de texto y correos electrónicos para sostener conversaciones). Las audiencias creativas son las que utilizan texto, audio y videos a fin de crear mensajes para grupos más grandes de personas. Por último, las audiencias absorbentes son las que gastan tiempo significativo buscando información, jugando, etcétera.

Picard concluye que la mayoría de las personas combinan roles pasivos con activos en el consumo, pero la mayor parte del tiempo utilizado en los medios tiende a ser de uso pasivo. Para este mismo autor, los grandes consumidores de información y noticias tienden a ser individuos activos política, social y económicamente, y son quienes buscan amplitud y profundidad de noticias e información desde distintas fuentes y plataformas.

\subsection{Capital social y la participación política y ciudadana en los medios que sirven para conocer mejor a la audien- cia universitaria}

Lacy y Bauer (2006) señalan que en el área académica de la economía social se incluye el concepto de capital social. Ambos autores explican cómo el hábito de las audiencias vinculado al concepto de capital social ayuda a comprender mejor su comportamiento respecto al consumo de contenidos informativos.

La esencia sobre el concepto de capital social puede definirse como el valor que se deriva de los recursos que se integran en una comunidad a través de los vínculos sociales con los demás (Lin, 2008). Por su parte, Gil de Zúñiga, Barnridge y Scherman (2017) señalan que el concepto de capital social puede estudiarse cuando se considera a la comunidad como conjunto de redes difusas de relaciones personales, en el que se identifica el valor que estas relaciones agregan a la vida de las personas en lo individual.

Respecto a lo anterior, puede observarse que las redes sociales en internet han contribuido a generar nuevas formas de conectar a las personas con la política, a través de cómo consumen las noticias e interactúan con las empresas informativas, y por la forma en que se expresan e involucran en las discusiones políticas a través de los medios de comunicación (Bachmann, Correa, y Gil de Zúñiga, 2012; Bakshy, Messing y Adamic, 2015; Barnidge, 2015; Gil de Zúñiga, Molyneux y Zheng, 2014; Barthel, Shearer, Gottfried y Mitchell, 2015; Kim, 2011; Valenzuela, 2013).

La participación política de una sociedad también puede medirse a través de diversos comportamientos políticos individuales. Por ejemplo, el votar, la actividad en la campaña política, el contacto con oficiales, y las diversas 
acciones sociales. Al respecto, Gil de Zúñiga, Veenstra, Vraga y Shah (2010) muestran que la participación política ciudadana a través de internet está identificada con razones personales $y$ que el comportamiento puede ser impulsado por las distintas identidades sociales y políticas de las personas. No obstante, la participación política a través de internet puede ser baja.

Existe una estrecha relación entre el consumo de noticias y uso de redes sociales en internet y el capital social (Bachmann et al., 2012; Howard, Savage, Flores-Saviaga, Toxtli y Monroy-Hernández, 2017). Hampton, Lee y Her (2011) demuestran que las redes sociales contribuyen a un comportamiento offline que genera capital social en la comunidad. Mientras que Gil de Zúñiga et al. (2017) evidencian la relación entre el capital social offline tradicionalmente conocido- y el capital social en redes sociales (social media-social capital) como un círculo virtuoso. No obstante, estos mismos autores concluyen que el comportamiento de social media-social capital de la comunidad predice el comportamiento de participación política offline de capital social de forma más intensa que la online.

Las audiencias de los medios de comunicación han sido estudiadas y comprendidas como grupos de ciudadanos que ejercen sus derechos de comunicación y participan en la esfera pública, así como grupos de consumidores de información y entretenimiento a través de los contenidos ofrecidos en los medios (Fuente-Cobo, 2014). En esta investigación se considera a la audiencia universitaria como el grupo de ciudadanos que se distinguen, además, por ser altos consumidores de información y entretenimiento a través de la oferta de la industria, siendo las redes sociales las plataformas más utilizadas y los dispositivos móviles los elegidos para comunicarse, informarse y entretenerse.

\section{Metodología}

El trabajo que aquí se presenta sigue el enfoque cuantitativo y tiene como principal objetivo identificar las características que distinguen a un grupo de la audiencia universitaria en México conforme a los principales parámetros del capital social y los medios. A su vez, interesa identificar el perfil de este grupo según los criterios de la segmentación de audiencias. La metodología utilizada es la del escalamiento Likert, para medir por escalas las variables que constituyen las actitudes de este grupo.

El contenido de la encuesta incluye preguntas para conocer el nivel de interés que tiene la comunidad universitaria hacia la política; en qué medida ha participado en actividades vinculadas con las instituciones $\mathrm{u}$ organizaciones comunitarias offline y online, e identificar qué medios de comunicación utiliza con mayor frecuencia para enterarse de las noticias e interactuar con los contenidos informativos. También interesa descubrir el nivel de confianza en las fuentes de información sobre asuntos de política y aquel que tiene en las instituciones. Importa decir que el cuestionario está integrado por 93 preguntas vinculadas a bloques específicos que tratan sobre estos temas. 
En esta investigación se entiende por la audiencia universitaria en México a los jóvenes adultos que participan activamente en la comunidad, ya sea como estudiantes o como profesores, y que representan a los estados de Jalisco, Aguascalientes y Ciudad de México. El periodo que se abrió para conocer la opinión de esta audiencia fue del 1 de agosto al 31 de diciembre de 2017. La encuesta fue contestada por 2,028 personas, de las cuales 1,195 terminaron la totalidad de las preguntas. El número de abandonos de los participantes pudo deberse al tiempo promedio que se llevaba en completar la encuesta que fue de 8 minutos y, quizá, por el número elevado de preguntas realizadas que fueron 93. Por tanto, este estudio tomó de muestra para la investigación a 1,195 personas. El promedio de edad fue de 23 años y el 96\% de la población encuestada tuvo acceso a internet a través de sus dispositivos móviles.

En el estudio se utilizó una probabilidad de acierto ( $p$ ) de 0.5 para determinar el tamaño de la muestra. El nivel de confianza utilizado fue de $95 \%$ y un error de estimación del 3.74\%. Esto dio un tamaño de la muestra de mínimo 707 personas, por lo que la muestra de 1,195 es estadísticamente aceptable.

Para la recopilación de datos se envió la encuesta en formato digital a tres campi universitarios en las ciudades de Aguascalientes, Guadalajara y Ciudad de México. Estas instituciones de educación superior son privadas. Los autores solicitaron apoyo a directores, coordinadores, profesores y alumnos para la distribución de la encuesta entre la comunidad universitaria de estos campi, misma que está integrada por cerca de 14,000 estudiantes de licenciatura y posgrados. Fueron seleccionadas las tres universidades de estos estados por el interés mostrado por los directivos hacia esta investigación y las facilidades otorgadas por ellos para la adecuada distribución de la encuesta.

Las hipótesis de partida que sirvieron para la elaboración de las preguntas de investigación fueron las siguientes:

1. El capital social es el valor que se deriva de los recursos que se integran en una comunidad a través de los vínculos con los demás. Los medios de comunicación tradicionales y digitales contribuyen a la generación de nuevas formas de conectar a las personas con la política, a través de su implicación y consumo de noticias; en las discusiones políticas o la participación activa en dichos medios. De aquí que la primera pregunta de investigación es: ¿Cuáles son las características que definen a esta audiencia universitaria en México según su participación política y cívica, y su participación interactiva en medios en asuntos relacionados a política y ciudadanía?

2. La confianza en las instituciones reduce la incertidumbre, estabiliza las relaciones sociales y agiliza las distintas transacciones sociales (Rinsdorf, 2017). Por su parte, Albarran (2017) señala que el consumidor tiene distintos antecedentes culturales que son únicos y que existen diferencias entre los consumidores en términos 
de edad, ciclo de vida y etnicidad, que contribuyen a explicar el por qué y cómo utilizan los medios. Por tanto, la segunda pregunta de investigación es: ¿Existen diferencias significativas del perfil de esta audiencia universitaria en México según el ciclo de edad y género respecto al nivel de confianza que tienen en las instituciones del país?

3. Picard (2014) establece una categoría de audiencias según sus hábitos de consumo y participación política medida a través de su interacción con los medios. De esta forma clasifica a las audiencias en pasivas, conservadoras, creadoras y absorbentes. Desde este argumento, la tercera pregunta de investigación es: ¿Puede definirse un perfil de esta audiencia universitaria en México según su interacción en asuntos comunitarios y de política en los medios de comunicación nacional desde la clasificación realizada por Picard (2014)?

Para el análisis de los resultados se cálculo la distribución F de Snedecor, que es una distribución de probabilidad continua y que se aplica para identificar la inferencia estadística. Esta técnica permite detectar la existencia o inexistencia de diferencias significativas entre muestras diferentes y que por la naturaleza de las preguntas de investigación se considera esencial.

\section{Resultados}

1. ¿Cuáles son las características que definen a esta audiencia universitaria en México según su participación polí- tica y cívica, y su participación interactiva en medios en asuntos relacionados a política y ciudadanía?

El $74 \%$ del total de los encuestados mostró interés en asuntos de política nacional. Su participación activa de contacto personal en instituciones $\mathrm{u}$ organizaciones civiles fue distinta a la que tuvieron en internet, es decir, esta audiencia mexicana prefirió el contacto personal y la cercanía respecto a la participación ciudadana que utilizar internet en aspectos comunitarios como donar dinero; participar en discusiones o debates con asuntos comunitarios; trabajar con otros para resolver problemas; participar en asuntos de servicio social, y contactar a un oficial público o agencia gubernamental. El Gráfico 1 muestra el porcentaje de participación en actividades comunitarias o caritativas a través de contacto personal y el uso de internet para estos fines.

Por otra parte, el 53.6\% de los entrevistados reconoció haber participado a través de internet desde 'algunas veces' hasta 'siempre', en asuntos comunitarios o de servicio social, siendo más frecuente 'algunas veces'.

Respecto a la disposición de donar dinero a una institución civil u organización caritativa, el 39\% indicó que estaría dispuesto a donar a través de internet sin importar de qué tipo de institución se trate. Por otro lado, el $52 \%$ de los encuestados contestó que sí donaría dinero offline. Es decir que esta audiencia universitaria prefirió hacer donativos de forma personal, probablemente porque no confía hacerlo en internet al desconocer a la 
institución. Se encontró una diferencia significativa con un $\mathrm{p}$-value $=$ 0.0000 y $\mathrm{Z}=-6.4163$ al comparar ambos porcentajes a un nivel de confian- za del 95\%. Esto confirma que fue mayor el porcentaje de personas que haría donativos directamente a las instituciones sin uso de internet.

Gráfico 1. Porcentaje de participación de esta audiencia universitaria en México según las actividades políticas y cívicas de forma offline versus online.

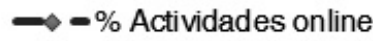

Donar dinero a una institución

civil u organización comunitaria o caritativa

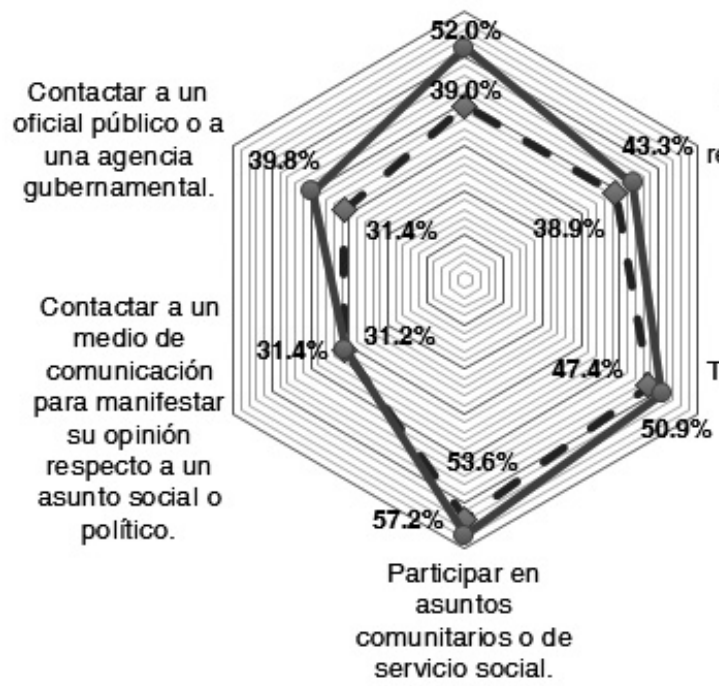

Participar en discusiones o debates relacionados con asuntos comunitarios.

\section{Trabajar con otros} para resolver problemas comunitarios.

Fuente: Elaboración propia.

El $38.9 \%$ del total de encuestados participó en discusiones o debates relacionados con asuntos comunitarios en línea, mientras que el $43.3 \%$ de la población encuestada participó de for- ma personal. La diferencia fue significativa entre la forma de participación ciudadana en internet y en persona. Por ejemplo, se tiene que $\mathrm{Z}=-2.2281$ y el p-value fue de 0.0132 al comparar 
el porcentaje de las personas que participó en debates sobre asuntos comunitarios. Por tanto, esta comunidad sí prefirió debatir personalmente en lugar de hacerlo online. Por último, también se encontró diferencia significativa entre las personas que prefirieron contactar a un oficial público o a una agencia gubernamental online y offline. El valor $\mathrm{p}$-value $=0.000$ y $\mathrm{Z}=-4.2545$ indican que fue mayor el porcentaje de personas que contactarían a un oficial público o agencia gubernamental en persona.

Finalmente, no se encontró diferencia significativa en la participación cívica online y offline de esta audiencia en los siguientes rubros: contactar a un medio de comunicación para expresar su opinión respecto a un asunto social o político, participar en asuntos comunitarios o de servicio social y trabajar con otros para resolver problemas comunitarios.

2. ¿Existen diferencias significativas del perfil de esta audiencia universitaria en México según el ciclo de edad y género respecto al nivel de confianza que tienen en las instituciones del país?

En promedio, esta audiencia universitaria no confió en diez de las trece instituciones que se le dieron a evaluar. Sólo tres de ellas tuvieron calificaciones superiores a seis. Éstas fueron las instituciones religiosas, las universidades y los medios de comunicación. La escala utilizada para calificar a las instituciones fue del 1 al 10, en la que 1 significaba "no puedo confiar del todo" y 10 significaba "puedo confiar mucho". La
Tabla 1 muestra la tabulación cruzada de valores medios según las calificaciones que los encuestados dieron a las instituciones y presenta también la valoración que hacen según mujeres y varones.

Existieron diferencias significativas entre los varones y las mujeres respecto a las calificaciones que dieron a algunas de las instituciones. Hubo diferencia significativa en aquellas otorgadas a las instituciones religiosas, gente de negocios, corporaciones, gobierno federal, congreso, departamento de policía y presidente. Las mujeres fueron más críticas al momento de evaluar a estas instituciones.

Por otro lado, los varones evaluaron de forma más positiva y tuvieron mayor índice de confianza en las instituciones religiosas, las universidades, los medios de comunicación, la gente de negocios y las corporaciones. Este grupo masculino dio un promedio aprobatorio a estas cinco instituciones mientras que el grupo femenino sólo aprobó a las instituciones religiosas.

Las instituciones que tuvieron calificaciones reprobatorias son gente de negocios, corporaciones, los bancos, la suprema corte, el gobierno local, estatal, federal, el congreso, el departamento de policía y el presidente. Las instituciones más bajas fueron los partidos políticos.

Las instituciones religiosas están aprobadas por todos los grupos de edad. Las universidades recibieron calificación aprobatoria por personas menores de 35 años y reprobatoria por personas de 35 años en adelante. Los 
medios de comunicación fueron reprobados por los menores de 25 años y aprobados por los demás grupos de edad. La gente de negocios sólo fue aprobada por el grupo de edad que va de 35 a antes de 45 años y reprobada por los demás grupos de edad. Todas las demás instituciones recibieron calificaciones reprobatorias en todos los gru- pos de edad. No obstante, sólo se encontró diferencia significativa entre las calificaciones asignadas al presidente y a los partidos políticos entre los distintos grupos de edad. El grupo de menos de 25 años fue el que dio la calificación más alta aun siendo reprobatoria en comparación con los otros grupos de edad.

Tabla 1. Tabulación cruzada de valores medios según las calificaciones que dio esta audiencia universitaria encuestada en México a las instituciones nacionales.

\begin{tabular}{|l|c|c|c|c|c|}
\hline \multicolumn{1}{|c|}{ Instituciones } & $\begin{array}{c}\text { Promedio de } \\
\text { toda la muestra }\end{array}$ & $\begin{array}{c}\text { Promedio } \\
\text { varones }\end{array}$ & $\begin{array}{c}\text { Promedio } \\
\text { mujeres }\end{array}$ & $\begin{array}{c}\mathbf{F} \text { de } \\
\text { Snedecor }\end{array}$ & p-value \\
\hline Instituciones Religiosas & 7.80 & 8.14 & 7.50 & 5.2989 & 0.0231 \\
\hline Universidades & 6.14 & 6.41 & 5.85 & 2.5712 & 0.1120 \\
\hline Medios de comunicación & 6.04 & 6.26 & 5.78 & 1.3314 & 0.2518 \\
\hline Gente de negocios & 5.82 & 6.33 & 5.38 & 8.6979 & 0.0037 \\
\hline Corporaciones & 5.62 & 6.06 & 5.23 & 6.4964 & 0.0120 \\
\hline Bancos & 4.86 & 5.08 & 4.62 & 1.9814 & 0.1625 \\
\hline La Suprema Corte de Justicia & 4.21 & 4.52 & 3.92 & 2.4034 & 0.1243 \\
\hline Gobierno Local & 3.60 & 3.81 & 3.40 & 1.2816 & 0.2608 \\
\hline Gobierno Estatal & 3.55 & 3.83 & 3.33 & 1.9668 & 0.1628 \\
\hline Gobierno Federal & 3.54 & 3.92 & 3.19 & 4.2644 & 0.0412 \\
\hline El Congreso & 3.48 & 3.90 & 3.15 & 4.6513 & 0.0325 \\
\hline Departamento de Policía & 3.34 & 3.71 & 3.02 & 4.7666 & 0.0305 \\
\hline Presidente & 3.29 & 3.77 & 2.89 & 5.9704 & 0.0160 \\
\hline Partidos Políticos & 2.64 & 2.96 & 2.39 & 3.8230 & 0.0523 \\
\hline
\end{tabular}

Fuente: Elaboración propia.

3. ¿Puede definirse un perfil de esta audiencia universitaria en México según su interacción en asuntos comunitarios y de política en los medios de comunicación nacional desde la clasificación realizada por Picard (2014)?

Esta audiencia universitaria en México tuvo rasgos de ser pasiva y conservadora respecto a su consumo infor- mativo y participación política en los medios de comunicación. El 84\% de las personas entrevistadas indicó que 'nunca' o 'rara vez' han contactado a un medio de comunicación para expresar su opinión respecto a un asunto social o político. Este aspecto puede reflejar cierta apatía de la audiencia universitaria para manifestar su opinión respecto a cuestiones sociales y políticas. 
Tabla 2. Evaluación que le dio esta audiencia universitaria encuestada en México a las instituciones según edad en 2017.

\begin{tabular}{|l|c|c|c|c|c|c|c|}
\hline \multicolumn{1}{|c|}{ Institución } & $\begin{array}{c}\text { Promedio } \\
\text { asignado por } \\
\text { todos los } \\
\text { participantes }\end{array}$ & $\begin{array}{c}\text { Menos } \\
\text { de 25 } \\
\text { años }\end{array}$ & $\begin{array}{c}\text { Desde } \\
\text { 25 hasta } \\
\text { antes de } \\
\text { 35 años }\end{array}$ & $\begin{array}{c}\text { Desde 35 } \\
\text { hasta } \\
\text { antes de } \\
\text { 45 años }\end{array}$ & $\begin{array}{c}\text { Igual o } \\
\text { mayor } \\
\text { de 45 } \\
\text { años }\end{array}$ & $\begin{array}{c}\text { F de } \\
\text { Snedecor }\end{array}$ & p-value \\
\hline $\begin{array}{l}\text { Instituciones } \\
\text { religiosas }\end{array}$ & 7.80 & 7.75 & 8.50 & 7.90 & 7.59 & 0.7479 & 0.5251 \\
\hline Universidades & 6.14 & 6.28 & 6.33 & 4.78 & 5.71 & 1.5951 & 0.1928 \\
\hline $\begin{array}{l}\text { Medios de } \\
\text { comunicación }\end{array}$ & 6.04 & 5.93 & 6.42 & 7.22 & 6.00 & 0.7624 & 0.5167 \\
\hline Gente de negocios & 5.82 & 5.87 & 5.83 & 6.00 & 5.29 & 0.4135 & 0.7454 \\
\hline Corporaciones & 5.62 & 5.69 & 5.55 & 5.80 & 5.00 & 0.5598 & 0.6423 \\
\hline Bancos & 4.86 & 4.81 & 5.67 & 5.10 & 4.53 & 0.8094 & 0.4904 \\
\hline $\begin{array}{l}\text { La suprema corte } \\
\text { de justicia }\end{array}$ & 4.21 & 4.32 & 4.25 & 4.33 & 3.29 & 0.9065 & 0.4394 \\
\hline Gobierno local & 3.60 & 3.65 & 3.75 & 3.89 & 3.00 & 0.4768 & 0.6989 \\
\hline Gobierno estatal & 3.55 & 3.67 & 4.33 & 3.00 & 2.41 & 2.3507 & 0.076 \\
\hline Gobierno federal & 3.54 & 3.72 & 3.42 & 2.70 & 2.76 & 1.4103 & 0.2418 \\
\hline El congreso & 3.48 & 3.73 & 2.92 & 3.00 & 2.29 & 2.6163 & 0.0541 \\
\hline $\begin{array}{l}\text { Departamento de } \\
\text { policía }\end{array}$ & 3.34 & 3.43 & 3.42 & 3.22 & 2.65 & 0.7853 & 0.5066 \\
\hline Presidente & 3.29 & 3.64 & 2.17 & 2.00 & 2.29 & 4.2007 & 0.0068 \\
\hline Partidos políticos & 2.64 & 2.86 & 2.33 & 1.70 & 1.69 & 3.2281 & 0.0247 \\
\hline
\end{tabular}

Fuente: Elaboración propia.

Por otra parte, el $76 \%$ de los participantes 'rara vez' o 'nunca' han respondido a un comentario en un periódico o blog respecto a un asunto social o político. Mientras que el $77 \%$ de las personas encuestadas señaló que 'rara vez' o 'nunca' ha contribuido con un artículo propio, fotografía o video, relacionado con un asunto social o político en los medios de comunicación.

\section{Conclusiones}

Esta investigación presentó el perfil de una audiencia universitaria con base a las respuestas que dieron 1,195 jóvenes adultos integrantes de la comunidad universitaria de tres instituciones de educación superior en Aguascalientes, Guadalajara y Ciudad de México.

El perfil de la audiencia fue medido según su participación cívica, interés en la política, interacción en los medios tradicionales y digitales, y por el nivel de confianza en las instituciones. Los resultados mostraron un bajo índice de capital social medido bajo estos parámetros. Esto puede explicarse no sólo por la cultura y las características sociales de la población, sino también por el alto 
consumo de productos de entretenimiento digital y la forma en cómo consumen la información ofrecida por las empresas informativas según los resultados mostrados en la investigación realizada por Padilla (2014), Leyva et al. (2015) y Gutiérrez-Rentería et al. (2016).

Por otro lado, los resultados de esta investigación también muestran que estos jóvenes adultos prefirieron una participación política en persona que a través del uso de los medios. Este resultado también coincide con el encontrado por Gutiérrez-Rentería et al. (2016) en el que el $71 \%$ de los jóvenes preferían manifestar sus opiniones y dialogar en persona a hacerlo a través del uso de medios. Finalmente, esta investigación también coincide con los hallazgos encontrados por Aguirre et al. (2016) respecto a lo que entienden los jóvenes por actividad cívica en internet.

Esta audiencia universitaria mexicana no confía en la mayoría de las trece instituciones que se le dieron a evaluar. Las instituciones que tuvieron índice aprobatorio por esta audiencia fueron las religiosas, las universidades y los medios de comunicación. Al igual que para Meneses et al. (2014), las universidades formaron parte de las instituciones más valoradas por los jóvenes adultos.

Por otro lado, el nivel de confianza en el sistema periodístico del país fue aprobatorio según la definición otorgada para este criterio de Rinsdorf (2017). Este aspecto es significativo dado que el estudio Digital News Report 2017 mostró que México ocupa el onceavo lugar de los países que más confían en las noticias según el ranking establecido por esa institución, y que incluyó una muestra de 36 países.

No obstante, los resultados de este estudio también evidencian la desaprobación de esta audiencia a las instituciones, las calificaciones fueron reprobatorias. Esto sirve como una llamada de alerta para las diferentes instituciones que más poder de influencia tienen en el país. Existe un mayor nivel de urgencia para atender la falta de confianza de los universitarios en los partidos políticos y el presidente.

Este estudio también presenta las diferencias significativas que existieron entre grupos de edad para evaluar a las instituciones mexicanas. En este sentido, también se evidencia la importancia de la segmentación de las audiencias en términos de edad, ciclo de vida, y sexo. De alguna forma, esto sirve para comprender mejor por qué y cómo utilizan los medios, a la vez que puede dar una mayor guía sobre cómo, a partir de estas características de este grupo de la audiencia universitaria en México, se puede generar capital social a través de los medios.

El canal de comunicación -offline y online- de esta audiencia varió de acuerdo a su interés y, por tanto, su interacción con los medios fue diferente. Este hallazgo puede ser relevante para las distintas instituciones, ya que pueden ser más eficientes y dirigir mejor sus estrategias de comunicación.

También se concluye que el género de la persona influye en la evaluación a las instituciones. Existe una diferencia significativa en cuanto al nivel crítico de las mujeres versus al que muestran los varones. 
Finalmente, esta audiencia universitaria en México tuvo rasgos de ser pasiva y conservadora respecto a su consumo informativo y participación política en los medios de comunicación según los parámetros utilizados por Picard (2014). Este público reflejó apatía para manifestar su opinión respecto a cuestiones sociales y políticas del país, mismos resultados que fueron encontrados por la investigación realizada por Meneses, Ortega y Urbina (2014).

\section{Limitaciones y futuras líneas de inves- tigación}

Esta investigación sólo muestra el perfil de una audiencia universitaria en México situada en tres estados del país.

Se juzga pertinente que las características que definieron a esta audiencia universitaria en México tendrían que ser estudiadas a mayor profundidad respecto a la idiosincrasia mexicana y latinoamericana tal como lo sugiere Albarran (2017), dado que este público tiene distintos antecedentes culturales que son únicos.
Existe la necesidad de profundizar sobre aspectos de la economía social, capital social y hábitos de las audiencias según el ciclo de vida y el género de las personas. Valdría la pena desarrollar una investigación en la que se identifique el hábito de las audiencias establecido por Lacy y Bauer (2006) y la forma de consumo de información en específico de esta audiencia universitaria medida bajos los parámetros de capital social y la fragmentación de las audiencias.

Para futuros estudios se recomienda utilizar la misma metodología y encuesta en otras audiencias en diversas regiones del país. También se sugiere hacer un estudio comparativo con otros países para identificar los perfiles de las audiencias universitarias medidas bajo los mismos parámetros aquí establecidos con el objetivo de identificar en qué medida los factores socio-culturales influyen en la construcción de capital social a través de los medios y a la generación de capital de marca para las empresas de comunicación.

\section{Bibliografía}

Aguirre, P., Casco, J. y Zavariz, A. (2016). Redes sociales de comunicación: del entretenimiento al activismo social. En Congreso Latinoamericano de Investigadores de la Comunicación, XIII, 2016. Sociedad del Conocimiento y Comunicación: Reflexiones críticas desde América Latina (pp. 319-325). México: ALAIC.

Albarran, A. (2017). The media economy. Segunda edición. Nueva York: Routledge.

Bachmann, I., Correa, T. y de Zúñiga, H. (2012). Profiling online political content Creators: Advancing the paths to democracy. International Journal of E-Politics, 3(4), 1-19. doi: 10.4018/jep.2012100101.

Bakshy, E., Messing, S. y Adamic, L. (2015). Exposure to ideologically diverse news and opinion on Facebook. Science, 348(6239), 1130-1132. doi: 10.1126/science.aaal 160.

Barnidge, M. (2015). The role of news in promoting political disagreement on social media. Computers in Human Behavior, 52, 211-218. doi.org/10.1016/j.chb.2015.06.011. 
Domínguez, F., López González, R. y Ortiz-Henderson, G. (2017). Redes sociales Digitales y participación política de jóvenes universitarios de México y Chile: Una revisión de estudios. Revista Latinoamericana de Ciencias de la Comunicación,14(26), 89-92.

Doyle, G. (2013). Understanding media economics. Segunda edición. Londres: Sage

Farías, A. (2014). Jóvenes universitarios y la construcción de ciudadanía a través de Facebook en el contexto michoacano. En Encuentro Nacional de AMIC, XXVI, 2014. La investigación de la comunicación ante el nuevo marco regulatorio de las telecomunicaciones y la radiodifusión en México (pp. 644-656). México: AMIC.

Fuente-Cobo, C. (2014). Active audiences in the regulation of the audiovisual media. Consumer versus citizen in Spain and Mexico. Comunicar, 22(43), 91-98.

Galindo, J. y González-Acosta, J. (2013). \#YoSoy132. La primera erupción visible. México: Global Talent University Press.

Gil de Zúñiga, H., Molyneux, L. y Zheng, P. (2014). Social media, political expression, and political participation: Panel analysis of lagged and concurrent relationships. Journal of Communication, 64(4), 612-634. doi:10.1111/jcom.12103.

Gil de Zúñiga, H., Veenstra, A., Vraga, E. y Shah, D. (2010). Digital democracy: Reimagining pathways to political participation. Journal of Information Technology E Politics, 7(1), 36-51. doi.org/10.1080/19331680903316742.

Gil de Zúñiga, H., Barnridge, M. y Scherman, A. (2017) Social media social capital, offline social capital, and citizenship: Exploring asymmetrical social capital effects, Political Communication, 34(1), 44-68. doi:10.1080/10584609.2016.1227000.

Gimpel, G. (2015). The future of video platforms: Key questionshaping the TV and video industry. The International Journal on Media Management, 17(1), 25-46. doi.org/10.1080/142 41277.2015.1014039.

Gutiérrez-Rentería, M. (2011) Estrategias de Grupo Televisa: del monopolio audiovisual a la competencia. Madrid: Editorial Académica Española.

Gutiérrez-Rentería, M. (2017). México Profile. En Newman, N.; Fletcher, R.; Kalogeropoulos, A., Levy, D. y Kleis-Nielsen, R. (Eds.). Digital News Report 2017 (pp. 111-113). Londres: Reuters Institute-Oxford University.

Gutiérrez-Rentería, M., Santana-Villegas, J. y Pérez-Ayala, M. (2016). Smartphone: usos y gratificaciones de los jóvenes en México en 2015. Palabra Clave, 20(1), 47-68. doi: 10.5294/ pacla.2017.20.1.3.

Hampton, K., Lee, C. y Her, E. (2011). How new media affords network diversity: Direct and mediated access to social capital through participation in local social settings. New Media \& Society, 13(7), 1031-1049. doi.org/10.1177/1461444810390342.

Havens, T. y Lotz, A. (2012). Understanding media industries. Nueva York: Oxford University Press.

Howard, P., Savage, S., Flores-Saviaga, C., Toxtli, C. y Monroy-Hernández, A. (2017). Redes sociales, participación ciudadana y la hipótesis del slacktivismo: Lecciones del caso de” El Bronco. Recuperado de: arXiv preprint arXiv:1710.03330.

Instituto Nacional de Estadística y Geografía (INEGI). Recuperado de: http://www.beta.inegi. org.mx/app/indicadores/?ind=1002000001\#divFV1002000001\#D1002000001 
Lacy, S. y Bauer, J. (2006). Future directions for media economics research. En Albarran, A., Chan-Olmsted, S. \& Wirth, M. (Eds.). Handbook of media management and economics (pp. 655674). Londres: Psychology Press.

Lin, N. (2008). A network theory of social capital. En Castiglione, D.; Van Deth, J. \& Wolleb, D. (Eds.). The handbook of social capital. (pp. 50-69). Nueva York: Oxford University Press.

Macías, A. (2014). The Music That is Here to Stay: New Rules in State-Society. Revolutionizing the Interaction between State and Citizens through Digital. En Edwards, S. y Santos, D. Revolutionizing the interactions betweentate and citizens through digital communications (pp. 145159). Estados Unidos: IGI Global.

Martínez, J. y Acosta, T. (2016). \#Yosoy132 y Facebook: Articulación de movimientos sociales en el ciberespacio. En Congreso Latinoamericano de Investigadores de la Comunicación, XIII, 2016. Sociedad del Conocimiento y Comunicación: Reflexiones críticas desde América Latina (pp. 91-97). México: ALAIC.

Meneses, M., Ortega, E. y Urbina, G. (2014). Jóvenes conectados y participación político ciudadana en el proceso electoral de México 2012. Revista Versión. Estudios de comunicación y política, 34, 71-92.

Organización para la Cooperación y el Desarrollo Económico (OCDE). Recuperado de: http://www.oecd.org/education/skills-beyond-school/EAG2017CN-Mexico-Spanish.pdf.

Padilla, M. (2014). Ciudadanía política en la red. Análisis de las prácticas políticas entre jóvenes universitarios. Comunicación y Sociedad, 21, 71-100.

Picard, R. (2014). Las industrias informativas: ¿Tienen futuro? Palabra Clave, 17(4), 10691096. doi:10.5294/pacla.2014.17.4.4.

Portillo, M. (2014). Mediaciones tecnocomunicativas, movilizaciones globales y disputas por la visibilidad en el espacio público. Análisis del surgimiento del \#YoSoy132. Revista Argumentos, (27)75, 173-190.

Reguillo, R. (2012, mayo). Reflexiones iniciales en torno a \#YoSoy 132. Magis. Recuperado de: http://www.magis.iteso.mx/redaccion/reflexiones-iniciales-en-torno-yosoy 132.

Rinsdorf, L. (2017). The role of trust in value networks for journalism in a convergent media environment. En Altmeppen, K., Hollifield, A. y van Loon, J. (Eds.), Value-oriented media management: Decision making between profit and responsibility (223-232). Alemania: Springer.

Sánchez-García, K. (2016). Sobre los derechos de las audiencias en México. Comunicación y sociedad, 27, 97-120.

Sylvie, G. (2008). Market analysis. En Sylvie, G.; Leblanc, J., Hollifield, A., Lacy, S. y Broadrick, A. (Eds.). Media management. A casebook approach. Cuarta edición (pp. 197-230). Nueva York: Lawrence Erlbaum Associates.

Thompson, J. (1995). The media and modernity: A social theory of the media. Estados Unidos: Stanford University Press. 